\title{
Fast text-only domain adaptation of RNN-transducer prediction network
}

\author{
Pylkkonen, Janne
}

ISCA

2021

Pylkkonen , J , Ukkonen , A , Kilpikoski , J , Tamminen , S \& Heikinheimo , H 2021 , Fast text-only domain adaptation of RNN-transducer prediction network . in 22nd Annual Conference of the International Speech Communication Association, INTERSPEECH 2021. Proceedings of the Annual Conference of the International Speech Communication Association, INTERSPEECH, vol. 2 , ISCA , Baixas , pp. 1882-1886, Annual Conference of the International Speech Communication Association , Brno , Czech Republic , 30/08/2021 . https://doi.org/10.21437

http://hdl.handle.net/10138/336639

https://doi.org/10.21437/Interspeech.2021-1191

unspecified

publishedVersion

Downloaded from Helda, University of Helsinki institutional repository.

This is an electronic reprint of the original article.

This reprint may differ from the original in pagination and typographic detail.

Please cite the original version. 


\title{
Fast Text-Only Domain Adaptation of RNN-Transducer Prediction Network
}

\author{
Janne Pylkkönen ${ }^{1}$, Antti Ukkonen ${ }^{1,2}$, Juho Kilpikoski ${ }^{1}$, Samu Tamminen ${ }^{1}$, Hannes Heikinheimo ${ }^{1}$ \\ ${ }^{1}$ Speechly, Finland \\ ${ }^{2}$ Department of Computer Science, University of Helsinki, Finland \\ firstnamedspeechly.com
}

\begin{abstract}
Adaption of end-to-end speech recognition systems to new tasks is known to be challenging. A number of solutions have been proposed which apply external language models with various fusion methods, possibly with a combination of two-pass decoding. Also TTS systems have been used to generate adaptation data for the end-to-end models. In this paper we show that RNN-transducer models can be effectively adapted to new domains using only small amounts of textual data. By taking advantage of model's inherent structure, where the prediction network is interpreted as a language model, we can apply fast adaptation to the model. Adapting the model avoids the need for complicated decoding time fusions and external language models. Using appropriate regularization, the prediction network can be adapted to new domains while still retaining good generalization capabilities. We show with multiple ASR evaluation tasks how this method can provide relative gains of $10-45 \%$ in target task WER. We also share insights how RNN-transducer prediction network performs as a language model.

Index Terms: automatic speech recognition, end-to-end models, RNN-transducer, adaptation, language model
\end{abstract}

\section{Introduction}

Over the recent years, the focus in automatic speech recognition research has shifted from hybrid models to end-to-end (E2E) systems. Traditional hybrid models consist of separate models for acoustic, language, and pronunciation [1,2], whereas E2E models integrate all of these into a single network $[3,4,5,6]$. The benefit of the hybrid models is that they can take advantage of different data sources, especially large amounts of text-only data. End-to-end models, on the other hand, are trained from matched speech and transcriptions, so their exposure to different language content is more limited.

A particularly interesting E2E architecture is the RNNtransducer (RNN-T) [3, 4], which provides state-of-the-art performance in a wide variety of streaming applications $[6,7]$. Despite being an E2E architecture, RNN-T lends itself for a compelling interpretation as having separate language and acoustic models. However, some recent research have concluded that such an interpretation may not always hold well [8]. Even though it is possible to initialize the RNN-T prediction network from a large text corpus, it has been unclear how much of the predictive power of the initial language model (LM) remains after the RNN-T has been trained with speech data.

To customize the E2E models for a particular domain, several methods have been proposed $[9,6,10,7]$, including application of external LMs, and using TTS-generated data to finetune the network. Fusion methods require changes to the model and/or decoding, whereas TTS-adaptation is a straightforward extension of model fine-tuning. One of the most applied adaptation methods is the shallow fusion $[9,6,11]$, where external

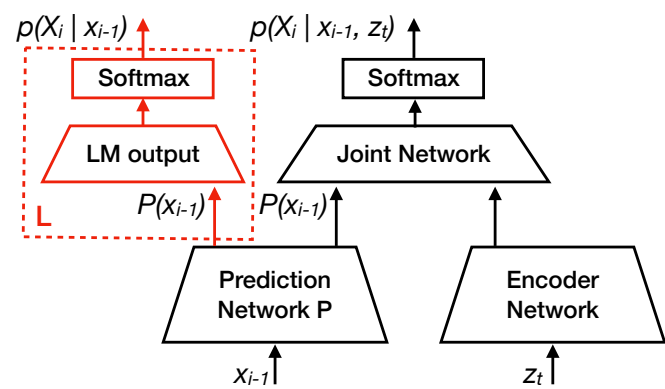

Figure 1: We propose a domain adaptation method for finetuning the prediction network $P$ of a trained RNN-T (shown in black). We first train a temporary LM output component denoted $L$ (shown in red) that enables the fine-tuning of $P$ with generic neural LM adaptation methods. Neither $L$ nor other changes to the RNN-T model are required when decoding.

language model scores are added to the RNN-T scores during decoding.

In this paper we present a simple yet effective RNN-T adaptation method, which requires only textual data. No speech data is involved in the adaptation process, not even via a TTS system. This text-only adaptation can be performed quickly, and it does not require any modifications to the decoding or model inference. The only additional requirement is the estimation of a temporary LM output layer on top of the prediction network (Fig. 1). With this output layer and suitable regularization, the prediction network can be adapted as a neural LM, while still remaining as a part of the RNN-T network. Using this RNN-T adaptation we observe 10-45\% relative word error rate (WER) improvements in target tasks. In contrast to shallow fusion, we show that these benefits can be obtained without substantial degradation in out-of-adaptation-domain accuracy.

Besides their practical applicability, our results also contribute to recent discussion about the role of the RNN-T prediction network $[8,11]$. In particular, we demonstrate that it is useful to interpret the RNN-T prediction network as having characteristics of an LM. This opens possibilities to using algorithms with E2E architectures that have previously only been applicable to hybrid models.

\section{Adaptation of RNN-T Prediction Network}

\subsection{RNN-T architecture}

The RNN-transducer, shown in black in Fig. 1, was first proposed by Graves [3, 4], and later refined by others [12, 6]. In RNN-T models both the prediction and encoder components are recurrent neural networks, typically LSTM stacks. Compared 
to simpler encoder networks trained with connectionist temporal classification (CTC) criterion [13, 14], RNN-T introduces a separate prediction network to condition the prediction of the next token $x_{i}$ to the past emissions $x_{1}, \ldots x_{i-1}$ in addition to the acoustic input $z_{t}$.

RNN-T is attractive especially when streaming decoding is required, as the decoding uses only the left context for predicting the next token. Streaming decoding requires that the recurrent hidden layers are implemented as uni-directional layers.

\subsection{Algorithm overview}

Our motivation for adapting a fully-trained RNN-T model is to quickly customize the network to different target tasks with text-only data. Moreover, we seek to do this without introducing further components to the model, and without increasing decoding complexity. In search of such a fast text-only domain adaptation method, we view the RNN-T prediction network as a neural language model.

Let $P$ denote the prediction network, $D_{t}$ the transcriptions used to train the input RNN-T, and $D_{a}$ the in-domain adaptation texts. At the core of our approach is to fine-tune the parameters of $P$ with $D_{a}$, just as one would adapt a neural LM in general. However, as part of an RNN-T model, the output of $P$, denoted $P(x)$, is an internal feature representation rather than a conditional probability distribution over the $i$ th token given the previous tokens, denoted $p\left(X_{i} \mid x_{i-1}\right)$, as required from an LM. Simple neural LM adaptation techniques cannot thus be applied directly to $P$, nor the RNN-T. We propose a two-step solution to this problem:

1. Pre-processing step With transcriptions in $D_{t}$, while keeping $P$ fixed, train a new LM output component to $P$ (red in Fig. 1) that outputs the conditional distribution $p\left(X_{i} \mid x_{i-1}\right)$. Let $L$ denote this LM output component.

2. Adaptation-step With transcriptions in $D_{a}$, fine-tune the LM induced by $P$ and $L$, but keep $L$ fixed. Output an RNN-T where $P$ replaces the original prediction network.

Note that the composition of $P$ and $L$ is a generic neural LM, and hence in the pre-processing step $L$ can be trained with text input using a standard autoregressive LM loss, such as cross-entropy. The training of $L$ is done over the transcriptions in $D_{t}$, to avoid introducing a mismatch with $P$ that was originally trained with those same transcriptions. With $L$ trained, we can proceed to the adaptation step in which we use the texts in $D_{a}$ to fine-tune the LM formed by $P$ and $L$.

Crucial to our approach is that when training $L$, the parameters of $P$ are fixed, and conversely, when fine-tuning $P$, the parameters of $L$ are frozen. This forces $L$ to first learn the same distribution of $P(x)$ that the RNN-T joint network expects, and then makes $L$ act as a regularizing constraint, preventing $P(x)$ from becoming incompatible with the joint network.

Note that $L$ is required only when fine-tuning $P$, it is not used during decoding. Overall, decoding is unaffected by this adaptation procedure, as no changes are introduced to the RNN$\mathrm{T}$ architecture. As the LM output component $L$ we chose to use a single feed-forward layer followed by a softmax, similar to the RNN-T joint network. In both steps, standard cross-entropy loss function was used for optimization.

\subsection{Regularization and optimization details}

We want to further ensure that $P$ does not change in ways that would be harmful for RNN-T decoding. To balance the fit to adaptation data $D_{a}$ and the transcriptions $D_{t}$ used during RNN$\mathrm{T}$ training, we propose to augment the cross-entropy loss used in the adaptation step with a term that penalizes changes in the predictions observed with common utterances. This additional regularization should promote the generalization ability of $P$.

To formalize this, denote the original, non-adapted prediction network with $P^{*}$, and let $x=\left(x_{0}, \ldots, x_{n}\right)$ denote an utterance with $n$ tokens, prepended with a start token $x_{0}$. Let $p\left(X_{i}\right.$ $\left.x_{i-1}\right)=L\left(P\left(x_{i-1}\right)\right)$ and $p^{*}\left(X_{i} \mid x_{i-1}\right)=L\left(P^{*}\left(x_{i-1}\right)\right) \mathrm{de}-$ note the distributions output by the LMs formed by $P$ and $L$ and $P^{*}$ and $L$, respectively, for input token $x_{i}$. The balancing loss term $\ell_{b}$ for input $x$ is then defined as

$$
\ell_{b}(x, P)=\frac{1}{n} \sum_{i=1}^{n} \operatorname{KLD}\left(p\left(X_{i} \mid x_{i-1}\right), p^{*}\left(X_{i} \mid x_{i-1}\right)\right),
$$

where KLD is the Kullback-Leibler divergence. The term $\ell_{b}(x, P)$ thus measures the difference between the next-token distributions induced by $P$ and $P^{*}$ for an utterance $x$.

To prevent $P$ from over-fitting to the adaptation corpus $D_{a}$, we use Eq.1 together with a set of utterances that are not part of $D_{a}$. We generate these utterances by sampling the LM induced by $P^{*}$. For each adaptation example $x$ in $D_{a}$, another utterance $\hat{x}$ of similar length is generated. Let $D_{b}$ denote the set of these utterances. We also introduce a second regularization term which penalizes the drifting of the weights of $P$ from their original values in $P^{*}$, defined as $\ell_{n}(P)=\left\|P-P^{*}\right\|_{2}$. The final adaptation loss function is hence:

$\ell(P)=\sum_{x \in D_{a}} \frac{\mathrm{CE}(x, P)}{\left|D_{a}\right|}+\frac{w_{b}}{\left|D_{b}\right|} \sum_{x \in D_{b}} \ell_{b}(x, P)+w_{n} \ell_{n}(P)$,

where $\mathrm{CE}(x, P)$ is the standard cross-entropy loss of the LM induced by $P$ for utterance $x$, and $w_{b}$ and $w_{n}$ are weights of the balancing and norm losses, respectively.

\section{Experiments}

\subsection{ASR system}

Our RNN-T architecture is similar to one presented by $\mathrm{He}$ et al. [6], utilizing layer-normalized LSTMs with projection layers. The first layer of the encoder network is a convolutional layer with 1536 filters. As input features we use 32 dimensional MEL filterbank energies, emitted 100 times a second. The convolutional layer reduces the frame rate to $30 \mathrm{~ms} /$ frame for the first two LSTM layers, after which the time reduction layer halves the frame rate to $60 \mathrm{~ms} /$ frame. There are $7 \mathrm{LSTM}$ layers in total, each with 1536 memory cells, and a projection dimension of 640 . For RNN-T training, the encoder network was initialized by training it first with a CTC loss function.

The prediction network consists of 2 layers of layernormalized LSTMs with projection layers, both with 1536 cells and a projection dimension of 640. It was initialized as a neural LM of the same architecture, augmented with a softmax output layer, and trained over a 20G-word subset of the English Oscar corpus [15]. During the initialization, the same word piece lexicon was used as with the joint network.

The joint network is a simple feed-forward network, which takes the inputs from the projection layers of the prediction and encoder networks. Softmax activation is applied to the joint network output, resulting in a 1001-dimensional output vector. The output encodes 1000 word pieces and a blank symbol. 
Table 1: Amount of adaptation (text) and evaluation (audio) utterances, and the size of the vocabulary in the datasets used in the experiments.

\begin{tabular}{lccc}
\hline Dataset & \#Adaptation utts & \#Eval utts & Vocabulary \\
\hline ATIS3 & 6355 & 965 & 1080 \\
Slurp & 10680 & 4173 & 5168 \\
Ted-lium & - & 1155 & 3652 \\
\hline
\end{tabular}

The training of the network was done with the RNN-T loss function until no error reduction over the training-time development set was observed. The network training was done with SGD using a slowly decaying learning rate. To reduce overfitting to the training data we applied SpecAugment [16] throughout the training.

During inference the RNN-T model was used with a beamsearch decoder, which restricts the maximum number of expanded hypotheses from frame-to-frame. For the experiments in this paper, this limit, the beam width, was set to 5 . For shallow fusion experiments, we converted an n-gram model into an FST, and added weighted LM scores to the RNN-T scores after each emission of a word piece.

\subsection{Data}

The RNN-T model was trained using three public English speech corpora: LibriSpeech [17], English Common Voice [18] (version 5.1, June 2020), and Ted-lium 3 [19]. We chose to use only utterances with durations in the $1-17 \mathrm{~s}$ range, resulting in $1.57 \mathrm{M}$, with total of about $2770 \mathrm{~h}$ of audio. During the training, a subset of Common Voice development set was used to monitor the progress and determine when to stop. The training ran about 20 epochs over the data.

The amount of speech training data is modest for an E2E model, and does not result in state-of-the-art results. However, we chose to use only well-known publicly available corpora to enable experiment reproducibility. We feel that the experiments remain informative and representative to the possible gains achievable by the proposed adaptation method. However, we decided to publish adaptation results also with our production model, which shares a similar RNN-T architecture, but has been trained with an order of magnitude more data.

The evaluations were carried out with three different datasets: Ted-lium 3 [19], ATIS3 [20], and Slurp [21]. For ATIS3 the evaluation used the December 1993 test set with the Crown microphone audios used where multiple audios were available. Adaptation used all unique transcriptions from the complete ATIS 3 training corpus. For Slurp the evaluation used the headset audio of each speaker for all test set transcriptions and for adaptation all the unique transcriptions from the nonsynthetic training set. The Slurp dataset is divided to 18 distinct real-life application scenarios, and the evaluations were carried out both using per-scenario subsets and using the scenarios pooled into one dataset. Table 1 summarizes the different evaluation sets. In all the experiments, the ASR accuracies were measured with word error rate.

The weights for the balancing and norm loss were optimized over the Slurp development set, where the model adaptation was carried out over the Slurp training transcriptions. This optimization showed that the adaptation method is not particularly sensitive to the exact values of the loss weights. All the experiments (except those in Section 3.4) were then carried out
Table 2: WER for unadapted and adapted models over evaluation corpora, and relative WER reduction.

\begin{tabular}{lccc}
\hline Dataset & Unadapted & Adapted & WERR-\% \\
\hline ATIS3 & $15.9 \%$ & $11.9 \%$ & $-25.2 \%$ \\
Slurp (pooled) & $42.8 \%$ & $38.6 \%$ & $-9.8 \%$ \\
Slurp (scenario) & $42.8 \%$ & $37.3 \%$ & $-12.9 \%$ \\
\hline ATIS3 (prod) & $9.7 \%$ & $5.4 \%$ & $-44.7 \%$ \\
Slurp (scen; prod) & $27.4 \%$ & $23.4 \%$ & $-14.6 \%$ \\
\hline
\end{tabular}
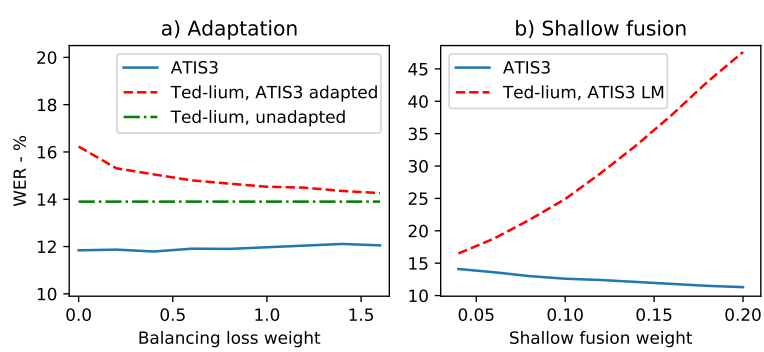

Figure 2: Adaptation experiments with in-domain and out-ofadaptation-domain evaluation. a) The effect of the balancing loss weight to the accuracy of the adapted models. b) Shallow fusion with ATIS3 4-gram, varying the shallow fusion weight.

with the same weights: $w_{b}=0.8, w_{n}=0.05$. The adaptation was stopped when the prediction network $L_{2}$-norm change exceeded value 4 .

\subsection{The effect of adaptation to ASR accuracy}

Table 2 shows the word error rates of unadapted and adapted models over two different datasets. For ATIS3 and Slurp evaluations, the models were adapted with dedicated held-out transcriptions. For Slurp, both the result for the pooled dataset and the overall result of per-scenario adaptations are shown. The results are shown with the experiment model described above, as well as our production model. With the experiment model, the adaptation ran for 16 and 23 epochs over the ATIS 3 and pooled Slurp adaptation data, respectively, until the maximum norm change condition was reached. The adaptation provides significant improvements in the accuracy, and it is achieved with remarkably small adaptation sets: only 6355 utterances for ATIS3, and 10680 utterances for Slurp.

\subsection{Generalization of the adapted models}

To show the effect of the balancing loss $\ell_{b}$ in Eq. 2, we used ATIS3 training data to adapt models with different balancing weights $w_{b}$. The resulting models were then tested both with ATIS 3 and Ted-lium 3 evaluation sets. Fig. 2 shows that accuracy on ATIS3 does not significantly vary with $w_{b}$. Ted-lium error rates, on the other hand, reduce as $w_{b}$ is increased.

Setting $w_{b}=0.8$, which was obtained by optimizing the adaptation weights over the Slurp development set, shows a good compromise between the adapted accuracy and generalization. With that value, Ted-lium WER degrades from the unadapted baseline by $5.5 \%$, but the ATIS 3 error reduced by $25 \%$. A larger balancing weight provides even better generalization: With value 1.6 Ted-lium results degrade only by $2.6 \%$ due to ATIS3 adaptation, while ATIS3 WER is still reduced by $24 \%$. 
Table 3: Word-level perplexities of held-out evaluation text corpora computed with the prediction network at different stages of RNN-T training and adaptation.

\begin{tabular}{lccc}
\hline & \multicolumn{3}{c}{ Perplexity } \\
Model & Oscar & LibriSp & ATIS3 \\
\hline \#1 Initializing LM & $\mathbf{1 2 3 . 6}$ & 286.7 & 238.4 \\
\#2 RNN-T, old LM output & 151.0 & 292.8 & 276.3 \\
\#3 RNN-T, new LM output & 179.8 & $\mathbf{2 3 1 . 4}$ & 261.9 \\
\#4 ATIS3 adapted RNN-T & 197.9 & 251.9 & $\mathbf{2 3 . 4}$ \\
\hline RNN-T, uninitialized $P$ & 1279.2 & 400.5 & 1137.0 \\
RNN-T, internal LM & 770.5 & 1116.9 & 1055.4 \\
\hline
\end{tabular}

\subsection{Comparison to shallow fusion}

Shallow fusion [9] has been shown to perform well with RNN-T models. It can be trained from a limited amount of text-data and hence applied easily to customize the ASR system for a target domain. However, it does require some changes to the decoder.

To study how shallow fusion compares to the presented RNN-T adaption method, we performed an evaluation with ATIS3 and Ted-lium 3 evaluation sets as in Section 3.4, but this time varying the shallow fusion weight. The shallow fusion LM was a 4-gram model over the word parts, trained from the ATIS3 adaptation set. The LM was smoothed with absolute discounting. The results in Fig. 2 show that shallow fusion improves the in-domain accuracy, as ATIS3 evaluation set WER drops to $11.1 \%$. However, this comes at a severe cost to the generalization: the accuracy on the Ted-lium set degrades drastically, even with small shallow fusion weights.

Finally we tested a combination of our RNN-T adaptation and shallow fusion. If the aim is to maximize the accuracy, without caring about the generalization, this combination provides the best results: Using ATIS3 adaptation set and the above mentioned 4-gram model provides a WER of $9.2 \%$ over the ATIS3 evaluation set.

\subsection{Prediction network as a language model}

The improvements obtained by the text-only adaptation of the prediction network $P$ suggest that it does indeed behave a lot like a language model. To analyze this characteristic further, we ran perplexity experiments with the prediction network and its LM output $L$ at different stages of the model training, namely the network intialization, RNN-T training, and adaptation. We used three held-out evaluations sets which match the data used in those stages: Oscar corpus subset, LibriSpeech test-clean transcripts, and ATIS3 adaptation set. Table 3 summarizes the results.

Model \#1 is the LM used to initialize $P$ before RNN-T training. It was trained from the Oscar corpus, so it is natural that it gives the lowest perplexities with the Oscar evaluation set. After the RNN-T training, but before the estimation of $L$, we obtain model $\# 2$, which reuses the feed-forward and softmax layers from \#1. Although the RNN-T training contained LibriSpeech data, we do not see improvements in the held-out LibriSpeech evaluation set perplexity, until $L$ is retrained with the speech transcripts (model \#3). However, the perplexity over the Oscar evaluation set degrades with the replacement of the LM output. Finally, after the adaptation with the ATIS3 training data (model \#4), we see a huge improvement in the corresponding evaluation set perplexity. For comparison, the ATIS3 evaluation set perplexity with a 4-gram model trained over the adaptation set with word piece segmentation was 39.7.

The conclusion from the perplexity experiments is that the prediction network $P$ and the LM output component $L$ together can perform well as a language model. During RNN-T training, $P$ learns to better predict the kind of utterances contained in the training data. However, to take the full advantage of the LM property of $P$ and $L$, a new LM output layer needs to be trained after the RNN-T training. This is because the LM output layer is analogous to the joint network, and during RNN-T training both the prediction network and the joint network can change in unison. Nevertheless, the fairly good perplexities for the model \#2 show that the representations of the trained $P$ are sufficiently close to the original so that even the output layer of the initializing LM works. This suggests that the initialization of the prediction network can have an important role. We verified this by training an RNN-T without initialization, as suggested by Ghodsi et al. [8]. The corresponding results in Table 3 show how the lack of a proper initialization greatly degrades the LM qualities of the prediction network. We found that the initialization not only helps in the LM task, but is beneficial also for ASR accuracy: without the initialization the WER over the Ted-lium 3 evaluation set degraded from $13.9 \%$ to $15.3 \%$.

A recent study [11] proposed that the internal language model of an E2E ASR model should be taken from the softmax output of the joint network. We have adopted a different view where the prediction network is used with a dedicated LM output layer, in the same way as it was initialized for the RNN-T training. For comparison, we tried the "internal LM" [11] approach, but the perplexities of the LM when taken through the joint network became much worse, see Table 3 .

\section{Conclusions}

In this paper we have presented a practical algorithm to perform domain adaptation of RNN-transducer E2E model with text-only data. The method is fast, requires no changes to the model inference, and works well even when very little data from the target domain is available. Compared to popular shallow fusion method, the presented RNN-T adaptation provides similar accuracy gains, but outperforms shallow fusion in the generalization capability. This can be contributed to our fully neural approach where the prediction network is modified under regularizing constraints.

The benefits of the RNN-T adaptation were shown with several evaluation tasks, using an experiment model which was trained from well-known public speech corpora, in the interest of experiment reproducibility. We also verified the adaptation gains with our production model, for which we have used an order of magnitude more training data. We further showed with LM perplexity experiments that simply by using a separate LM output layer, the prediction network provides a reasonable performance as a language model. Our evidence of the improvements obtained from adapting the prediction network lead us to conclude that the LM interpretation of the prediction network is not only justified, but also practical.

\section{Acknowledgements}

We thank Business Finland and CSC - IT Center for Science Ltd. for providing the supercomputer resources used to carry out the experiments in this paper. We also want to thank the Speechly developer community and customers for motivating us to continuously push the boundaries of our work. 


\section{References}

[1] G. Hinton, L. Deng, D. Yu, G. E. Dahl, A. Mohamed, N. Jaitly, A. Senior, V. Vanhoucke, P. Nguyen, T. N. Sainath, and B. Kingsbury, "Deep neural networks for acoustic modeling in speech recognition: The shared views of four research groups," IEEE Signal Processing Magazine, vol. 29, no. 6, pp. 82-97, 2012.

[2] F. Zhang, Y. Wang, X. Zhang, C. Liu, Y. Saraf, and G. Zweig, "Faster, Simpler and More Accurate Hybrid ASR Systems Using Wordpieces," in Proc. Interspeech 2020, 2020, pp. 976-980 [Online]. Available: http://dx.doi.org/10.21437/Interspeech.20201995

[3] A. Graves, "Sequence transduction with recurrent neural networks," CoRR, vol. abs/1211.3711, 2012. [Online]. Available: http://arxiv.org/abs/1211.3711

[4] A. Graves, A. Mohamed, and G. Hinton, "Speech recognition with deep recurrent neural networks," in 2013 IEEE International Conference on Acoustics, Speech and Signal Processing, 2013, pp. 6645-6649.

[5] W. Chan, N. Jaitly, Q. V. Le, and O. Vinyals, "Listen, attend and spell: A neural network for large vocabulary conversational speech recognition," in ICASSP, 2016.

[6] Y. He, T. N. Sainath, R. Prabhavalkar, I. McGraw, R. Alvarez, D. Zhao, D. Rybach, A. Kannan, Y. Wu, R. Pang, Q. Liang, D. Bhatia, Y. Shangguan, B. Li, G. Pundak, K. C. Sim, T. Bagby, S. Chang, K. Rao, and A. Gruenstein, "Streaming end-to-end speech recognition for mobile devices," in ICASSP 2019 - 2019 IEEE International Conference on Acoustics, Speech and Signal Processing (ICASSP), 2019, pp. 6381-6385.

[7] J. Li, R. Zhao, Z. Meng, Y. Liu, W. Wei, S. Parthasarathy, V. Mazalov, Z. Wang, L. He, S. Zhao, and Y. Gong, "Developing RNN-T Models Surpassing High-Performance Hybrid Models with Customization Capability," in Proc. Interspeech 2020, 2020, pp. 3590-3594. [Online]. Available: http://dx.doi.org/10.21437/Interspeech.2020-3016

[8] M. Ghodsi, X. Liu, J. Apfel, R. Cabrera, and E. Weinstein, "Rnntransducer with stateless prediction network," in ICASSP 2020 2020 IEEE International Conference on Acoustics, Speech and Signal Processing (ICASSP), 2020, pp. 7049-7053.

[9] A. Kannan, Y. Wu, P. Nguyen, T. N. Sainath, Z. Chen, and R. Prabhavalkar, "An analysis of incorporating an external language model into a sequence-to-sequence model," in 2018 IEEE International Conference on Acoustics, Speech and Signal Processing (ICASSP), 2018, pp. 1-5828.

[10] C. Shan, C. Weng, G. Wang, D. Su, M. Luo, D. Yu, and L. Xie, "Component fusion: Learning replaceable language model component for end-to-end speech recognition system," in ICASSP 2019 - 2019 IEEE International Conference on Acoustics, Speech and Signal Processing (ICASSP), 2019, pp. 5361-5635.

[11] Z. Meng, S. Parthasarathy, E. Sun, Y. Gaur, N. Kanda, L. Lu, X. Chen, R. Zhao, J. Li, and Y. Gong, "Internal language model estimation for domain-adaptive end-to-end speech recognition," in Proceedings of IEEE SLT, 2021.

[12] K. Rao, H. Sak, and R. Prabhavalkar, "Exploring architectures, data and units for streaming end-to-end speech recognition with rnn-transducer," in 2017 IEEE Automatic Speech Recognition and Understanding Workshop (ASRU), 2017, pp. 193-199.

[13] A. Graves, S. Fernández, F. Gomez, and J. Schmidhuber, "Connectionist temporal classification: Labelling unsegmented sequence data with recurrent neural networks," in Proceedings of the 23rd International Conference on Machine Learning, ser. ICML '06. New York, NY, USA: Association for Computing Machinery, 2006, p. 369-376. [Online]. Available: https://doi.org/10.1145/1143844.1143891

[14] A. Hannun, C. Case, J. Casper, B. Catanzaro, G. Diamos, E. Elsen, R. Prenger, S. Satheesh, S. Sengupta, A. Coates, and A. Y. Ng, "Deep speech: Scaling up end-to-end speech recognition," 2014
[15] P. J. Ortiz Suárez, B. Sagot, and L. Romary, "Asynchronous Pipeline for Processing Huge Corpora on Medium to Low Resource Infrastructures," in 7th Workshop on the Challenges in the Management of Large Corpora (CMLC-7), P. Bański, A. Barbaresi, H. Biber, E. Breiteneder, S. Clematide, M. Kupietz, H. Lüngen, and C. Iliadi, Eds. Cardiff, United Kingdom: Leibniz-Institut für Deutsche Sprache, Jul. 2019. [Online]. Available: https://hal.inria.fr/hal-02148693

[16] D. S. Park, W. Chan, Y. Zhang, C.-C. Chiu, B. Zoph, E. D. Cubuk, and Q. V. Le, "SpecAugment: A Simple Data Augmentation Method for Automatic Speech Recognition," in Proc. Interspeech 2019, 2019, pp. 2613-2617. [Online]. Available: http://dx.doi.org/10.21437/Interspeech.2019-2680

[17] V. Panayotov, G. Chen, D. Povey, and S. Khudanpur, "Librispeech: An asr corpus based on public domain audio books," in 2015 IEEE International Conference on Acoustics, Speech and Signal Processing (ICASSP), 2015, pp. 5206-5210.

[18] R. Ardila, M. Branson, K. Davis, M. Kohler, J. Meyer, M. Henretty, R. Morais, L. Saunders, F. Tyers, and G. Weber, "Common voice: A massively-multilingual speech corpus," in Proceedings of the 12th Language Resources and Evaluation Conference. Marseille, France: European Language Resources Association, May 2020, pp. 4218-4222. [Online]. Available: https://www.aclweb.org/anthology/2020.1rec-1.520

[19] F. Hernandez, V. Nguyen, S. Ghannay, N. Tomashenko, and Y. Estève, "Ted-lium 3: Twice as much data and corpus repartition for experiments on speaker adaptation," in Speech and Computer, A. Karpov, O. Jokisch, and R. Potapova, Eds. Cham: Springer International Publishing, 2018, pp. 198-208.

[20] D. A. Dahl, M. Bates, M. Brown, W. Fisher, K. Hunicke-Smith, D. Pallett, C. Pao, A. Rudnicky, and E. Shriberg, "Expanding the scope of the ATIS task: The ATIS-3 corpus," in Human Language Technology: Proceedings of a Workshop held at Plainsboro, New Jersey, March 8-11, 1994, 1994. [Online]. Available: https://www.aclweb.org/anthology/H94-1010

[21] E. Bastianelli, A. Vanzo, P. Swietojanski, and V. Rieser, "SLURP: A spoken language understanding resource package," in Proceedings of the 2020 Conference on Empirical Methods in Natural Language Processing (EMNLP). Online: Association for Computational Linguistics, Nov. 2020, pp. 7252-7262. [Online]. Available: https://www.aclweb.org/anthology/2020.emnlpmain. 588 